\title{
Epidemiology and risk factors of methicillin-resistant Staphylococcus aureus and vancomycin-resistant enterococci infections in Zhejiang China from 2015 to 2017
}

Lin Huang ${ }^{1}$, Rong Zhang ${ }^{1}$, Yanyan Hu${ }^{1}$, Hongwei Zhou' ${ }^{1}$ Junmin Cao ${ }^{2}$, Huoyang Lv ${ }^{3}$, Shi Chen ${ }^{4}$, Shibiao Ding ${ }^{5}$ and Gongxiang Chen ${ }^{*^{*}}$ (D)

\begin{abstract}
Background: Gram-positive bacteria are dangerous and challenging agents of infection due to their increasing resistance to antibiotics. We aim to analyse the epidemiology and risk factors of methicillin-resistant Staphylococcus aureus (MRSA) and vancomycin-resistant enterococci (VRE) in Zhejiang China.

Methods: Gram-positive bacteria (including S. aureus, Enterococcus faecalis and Enterococcus faecium) were collected from eighty-six hospitals of eleven cities in Zhejiang China from 2015 to 2017. The detection rates of MRSA and VRE infection were calculated for the non-duplicated isolate according to year, region, hospital level, patient age, specimen type and patient category. Meanwhile, the detected resistances of MRSA, E. faecalis and E. faecium to different antibiotics from 2015 to 2017 were compared. The risk factors and the differences in MRSA and VRE detection rates were compared using odds ratio (OR) with 95\% confidence interval (95\% Cl) and Chi-square test respectively.

Results: From 2015 to 2017, the detection rates of MRSA and VRE decreased gradually. The cities with the highest MRSA and VRE detection rates tended to be adjacent; for example, the neighbouring cities Hangzhou and Quzhou had simultaneously high rates of MRSA and VRE infection. Patients from IIIA hospital who were older than 75 years and in the intensive care unit (ICU) were most at risk. No vancomycin-resistant isolate was found in MRSA. Resistance of $E$. faecalis and E. faecium to vancomycin and linezolid decreased slightly and then maintained a low level.
\end{abstract}

Conclusions: The detection rates of MRSA and VRE stayed at moderate and low levels during the three year period of this study, while local dissemination was found in MRSA and VRE isolates. Sustained surveillance is necessary to prevent the spread or clonal dissemination of drug-resistant strains in Zhejiang China.

Keywords: Epidemiology, Risk factor, Methicillin-resistant Staphylococcus aureus, Vancomycin-resistant enterococci

\footnotetext{
* Correspondence: chengongxiang@zju.edu.cn

${ }^{1}$ Department of Clinical Microbiology, The Second Affiliated Hospital of

Zhejiang University School of Medicine, 88 Jiefang Road, Hangzhou, Zhejiang

310009, People's Republic of China

Full list of author information is available at the end of the article
}

(c) The Author(s). 2019 Open Access This article is distributed under the terms of the Creative Commons Attribution 4.0 International License (http://creativecommons.org/licenses/by/4.0/), which permits unrestricted use, distribution, and reproduction in any medium, provided you give appropriate credit to the original author(s) and the source, provide a link to the Creative Commons license, and indicate if changes were made. The Creative Commons Public Domain Dedication waiver (http://creativecommons.org/publicdomain/zero/1.0/) applies to the data made available in this article, unless otherwise stated. 


\section{Background}

Gram-positive bacteria, such as Staphylococcus aureus, Enterococcus faecalis, and Enterococcus faecium are dangerous and challenging agents of infection due to their increasing resistance to antibiotics [1]. Among these, $S$. aureus are the most frequently isolated, making up 29.1\% of the isolated Gram-positive bacterial populations, and infections caused by $S$. aureus (both community-associated or nosocomial-associated) are reported all over the world [2, 3]. Enterococci (including E. faecalis and E. faecium) are the second frequently isolated Gram-positive bacteria at $19.5 \%$. Past generations of enterococci were mainly associated to urinary-tract infection, but recently more and more enterococci are isolated from other infections [4], and an outbreak of vancomycin resistant enterococci (VRE) in a solid organ transplant unit was reported in 2018 [5].

The epidemiology of resistance in Gram-positive bacteria has undergone major changes in recent decades, with methicillin-resistant $S$. aureus (MRSA) and VRE now being of international concern [6]. Antimicrobial resistances of Gram-positive bacteria have been reported in many countries [7-9]; however, it was only in recent years that vanM-carrying E. faecalis strains were isolated from patients and the clinical environment in the Second Affiliated Hospital of Zhejiang University School of Medicine [10], we speculate that if vanM infection has been spreading in the Zhejiang province, it may lead to an increase of VRE overall. In China, although several local studies have examined the prevalence of resistance in Gram-positive bacteria [11, 12], the studies usually only cover the tertiary hospitals. Therefore, the present study is a retrospective surveillance covering a wider range of hospitals, including tertiary and secondary hospitals. Our aim is to seek trends in antimicrobial resistance among clinical isolates of important Gram-positive bacteria in the Zhejiang province of China.

\section{Materials and methods}

\section{Bacterial isolates}

We extracted data of $S$. aureus, E. faecalis and E. faecium infections from the Zhejiang surveillance system's outpatient and inpatient records from January 1, 2015 to December 31, 2017. The number of surveyed hospitals for each city was as follows: Hangzhou $(n=24)$, Huzhou $(n=4)$, Jiaxing $(n=11)$, Jinhua $(n=9)$, Lishui $(n=6)$, Ningbo $(n=10)$, Quzhou $(n=5)$, Shaoxing $(n=6)$, Taizhou $(n=5)$, Wenzhou $(n=5)$, and Zhoushan $(n=1)$. Sample collection was in accordance with the clinical microbiology manual [13]. To avoid duplicate isolates, only one isolate from the same species was included per patient, as determined by the personal identifying code and hospital name. Species identification of the isolates was performed by standard biochemical methods, automated system (the Vitek 2 compact, BD Phoenix-100, MicroScan WalkAway-96) or Matrix-Assisted Laser Desorption/Ionization Time of Flight Mass Spectrometry (MALDI-TOF-MS).

\section{Antimicrobial susceptibility testing}

Standard operation procedures were established according to the Clinical and Laboratory Standards Institute's (CLSI) criteria, M100-S24 [14]. Every participating laboratory conducted the antibiotic susceptibility testing for the clinical isolates using the Kirby-Bauer disk diffusion method or the commercialized automated system following the instrument specifications. S. aureus (ATCC 25923, ATCC 43300) and E. faecalis (ATCC 29212, ATCC 51299) were used as quality control strains for the testing.

\section{Statistical analysis}

We analysed the risk factors for MRSA and VRE proportions, and for the multivariable model, year, region, hospital level, patient age, specimen type and patient category were considered. The lowest detection rates of MRSA and VRE were set as contrasts. The risk factors and the differences in MRSA and VRE detection rates were compared using odds ratio (OR) with 95\% confidence interval $(95 \% \mathrm{CI})$ and Chi-square test. Chi-square values were corrected when the quantities of VRE isolates were less than forty. Results with $P$-value $<0.05$ were considered statistically significant. SPSS 19.0 (IBM Company, Chicago, IL) and WHONET 5.5 (WHO) software were used for all statistical analyses.

\section{Results}

Distribution of MRSA, Enterococci and VRE isolates

During the years 2015-2017, the total number of MRSA isolates increased from 9292 to 10,237 cases; in contrast, VRE isolates decreased from 173 to 137 cases (data not shown). Among the 11 cities that participated in the survey, Hangzhou contributed $37.6 \%$ of all MRSA isolates (29,866 cases); and $41.0 \%$ of all Enterococci isolates (58,329 cases), although the population of Hangzhou is only about 9.5 millon (16.5\% of the Zhejiang province population). This high number of isolates may be due to Hangzhou having more IIIA hospitals, which were the contributors of $67 \%$ of all MRSA and enterococci isolates. However, Hangzhou also contributed 245 VRE isolates, which was $54.2 \%$ of all of the VRE isolates in the study, and a much higher percentage than the province's MRSA and enterococci contributions. MRSA were most frequently isolated from respiratory samples $(14,339$ or $48.0 \%$ of all samples); VRE were dominantly isolated from urine samples (313 or $69.2 \%$ of all samples) (data not shown). The number of samples based on age of patient and inpatient vs. out-patient were also categorized. 
Patients older than 75 and patients who were hospitalized (especially those in the ICU) had dominantly higher MRSA, enterococci and VRE isolation rates compared to other patient groups (data not shown).

\section{Resistance rates of MRSA, E. faecalis and E. faecium to antimicrobial agents from 2015 to 2017}

During the period of 2015 to 2017, the resistance rates of MRSA to trimethoprim-sulfamethoxazole and nitrofurantoin increased from 9.66 to $14.94 \%(P<0.001)$ and from 0.51 to $0.95 \%(P<0.001)$ respectively. The resistance rates of MRSA to erythromycin, tetracycline, tigecycline and levofloxacin decreased from 90.32 to $88.24 \%(P=0.003)$, from 35.58 to $29.05 \%(P<0.001)$, from $0.14 \%$ to 0 , and from 53.01 to $40.77 \%(P<0.001)$ respectively (Fig. 1$)$. MRSA showed $100 \%$ susceptibility to vancomycin and teicoplanin, however, a few linezolid-resistant strains were found in 2016. The resistance rates of E. faecium to nitrofurantoin and gentamicin-high level increased from 51.90 to $55.47 \%$ $(P<0.001)$ and from 34.15 to $41.67 \%(P<0.001)$ increased respectively. The resistance rates of $E$. faecium to linezolid, tetracycline, teicoplanin and vancomycin decreased from 1.15 to $0.67 \%(p=0.168)$, from 38.33 to $30.77 \%(p=0.243)$, from 0.51 to $0.16 \%(P<0.001)$, and from 1.69 to $0.90 \%$ ( $p=$ $0.006)$ respectively (Table 1). The resistance rate of $E$. faecalis to gentamicin-high level increased from 7.32 to $41.67 \%$ $(P<0.001)$. The resistance rates of $E$. faecalis to ampicillin, nitrofurantoin, linezolid, tetracycline, and levofloxacin decreased from 5.72 to $3.04 \%$ ( $p=0.021$ ), from 3.95 to $2.56 \%$ $(P<0.001)$, from 3.17 to $2.73 \%(p=0.002)$, from 77.29 to $76.32 \%(P<0.001)$, and from 20.78 to $19.79 \%(p=0.009)$ respectively (Table 1). Tigecycline showed $100 \%$ susceptibility against $E$. faecalis and $E$. faecium. The resistance rates of $E$. faecium to ampicillin, nitrofurantoin, ciprofloxacin, gentamicin-high level, levofloxacin, teicoplanin and vancomycin were higher than that of $E$. faecalis, while lower resistance rates to tetracycline and linezolid were seen in $E$. faecium in comparison to E. faecalis.

\section{Resistance rates of S. aureus and Enterococci to antimicrobial agents among different patients}

For isolates collected from the outpatients, non-ICU inpatients and ICU inpatients, resistance rates of $S$. aureus to all the antimicrobial agents increased gradually except for clindamycin and trimethoprim-sulfamethoxazole (Fig. 2). Among which, the resistance rates to oxacillin, gentamicin and levofloxacin increased more dramatically. Resistance rates of enterococcus (including E. faecalis and E. faecium) to all the antimicrobial agents increased gradually except for linezolid and tetracycline (Fig. 3).

\section{Analysis of risk factors associated with MRSA and VRE strains}

In multivariable analysis for risk factors associated with MRSA and VRE (Table 2), we observed that the 2015 isolation rates of MRSA (OR $=1.022$, 95\% CI 1.010 $1.035, p<0.001)$ and of VRE (OR $=1.005$, 95\% CI 1.003$1.006, p<0.001)$ were higher than those in 2017. Compared to Huzhou, Zhoushan was the most likely city to have MRSA isolates $(\mathrm{OR}=1.775,95 \%$ CI $1.676-1.880$, $p<0.001)$. Compared to Lishui, Huzhou was the most likely city to have VRE isolates $(\mathrm{OR}=1.025,95 \% \mathrm{CI}$ 1.017-1.033, $p<0.001)$. The cities with high detection rates of MRSA were distributed in the east and west of

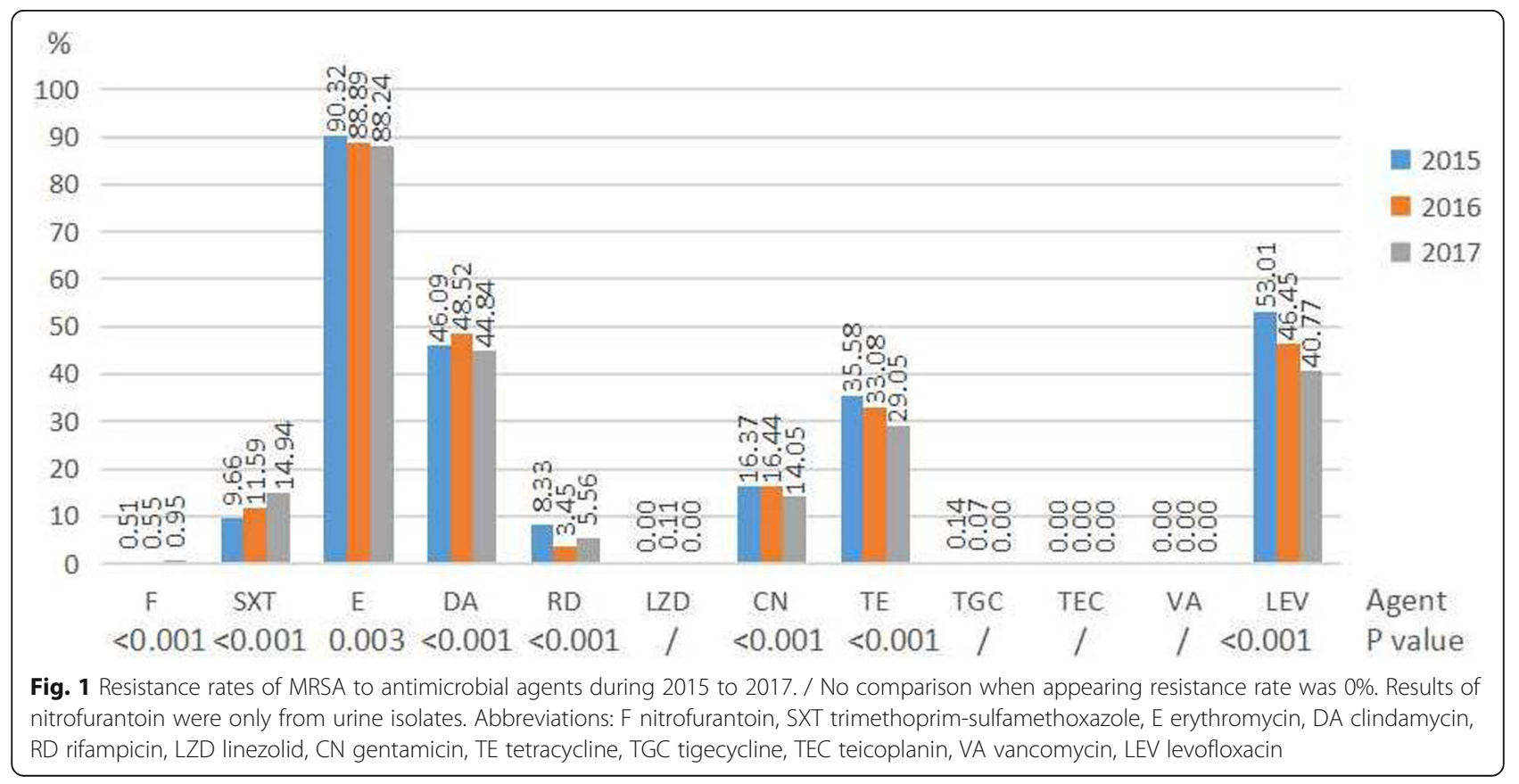


Table 1 Resistance rates (\%) of E. faecalis (E.fa) and E. faecium (E.fm) to antimicrobial agents during 2015 to 2017

\begin{tabular}{|c|c|c|c|c|c|c|c|c|}
\hline \multirow{2}{*}{$\begin{array}{l}\text { Antimicrobial } \\
\text { agent }\end{array}$} & \multicolumn{2}{|l|}{2015} & \multicolumn{2}{|l|}{2016} & \multicolumn{2}{|l|}{2017} & \multicolumn{2}{|l|}{$P$ value } \\
\hline & $\mathrm{E} . \mathrm{fa}(n=9109)$ & E.fm $(n=7106)$ & $\mathrm{E} . \mathrm{fa}(n=10,740)$ & E.fm $(n=9135)$ & E.fa $(n=11,860)$ & E.fm $(n=10,379)$ & E.fa & E.fm \\
\hline Ampicillin & 5.72 & 88.22 & 4.66 & 89.58 & 3.04 & 89.13 & 0.021 & $<0.001$ \\
\hline Nitrofurantoin & 3.95 & 51.90 & 3.19 & 54.96 & 2.56 & 55.47 & $<0.001$ & $<0.001$ \\
\hline Gentamicin-High & 7.32 & 34.15 & 18.06 & 41.57 & 41.67 & 41.67 & $<0.001$ & $<0.001$ \\
\hline Ciprofloxacin & 22.54 & 89.13 & 23.14 & 90.47 & 22.05 & 90.32 & 0.009 & 0.147 \\
\hline Linezolid & 3.17 & 1.15 & 2.97 & 1.00 & 2.73 & 0.67 & 0.002 & 0.168 \\
\hline Tetracycline & 77.29 & 38.33 & 76.90 & 33.75 & 76.32 & 30.77 & $<0.001$ & 0.243 \\
\hline Tigecycline & 0 & 0 & 0 & 0 & 0 & 0 & / & / \\
\hline Teicoplanin & 0 & 0.51 & 0 & 0.45 & 0 & 0.16 & / & $<0.001$ \\
\hline Vancomycin & 0.67 & 1.69 & 0.37 & 1.22 & 0.42 & 0.90 & $<0.001$ & 0.006 \\
\hline Levofloxacin & 20.78 & 87.49 & 20.43 & 89.04 & 19.79 & 88.47 & 0.009 & 0.195 \\
\hline
\end{tabular}

/ No comparison when appearing resistance rate was $0 \%$

the Zhejiang province, and the cities with high detection rates of VRE were distributed in the west of the Zhejiang province (Fig. 4). Isolates from tertiary hospitals were more likely to be MRSA and VRE than isolates from secondary hospitals. Analysis based on age groups revealed that isolates derived from patients older than 75 years had the highest proportion of MRSA (OR $=1.443$, 95\% CI 1.409-1.478, $p<0.001)$ and VRE (OR $=1.011,95 \% \mathrm{CI}$ $1.009-1.013, p<0.001)$. Isolates from bile had the highest proportion of MRSA (OR $=1.385$, 95\% CI 1.151$1.665, p<0.001)$ and isolates from blood had the highest proportion of VRE $(\mathrm{OR}=1.007,95 \%$ CI 1.003-1.010, $p<0.001)$. Patients who were in ICU had the highest proportion of MRSA (OR $=1.439,95 \%$ CI $1.407-1.473$, $p<0.001)$ and VRE $(\mathrm{OR}=1.019,95 \%$ CI $1.013-1.025$, $p<0.001)$.

\section{Discussion}

It is a global trend that the drug resistance of Gram-positive bacteria decreases gradually. In our study, there is a slight decline in the isolation rates of MRSA (from 34.98 to $33.53 \%$ ). The downward trend is also observed in France, Germany and the UK $[15,16]$, but it is not seen in Saudi Arabia, where MRSA is maintaining its high level [17]. The use of alcohol-based hand-rub and decolonization with antimicrobial agents may have helped to reduce MRSA transmission. As with other studies, linezolid, tigecycline and vancomycin are the most active agents against MRSA [18]. There were no MRSA isolates that were resistant to vancomycin and teicoplanin. Unlike other reports, the minimum inhibitory concentration (MIC) of MRSA to vancomycin was distributed around 0.5 and 1 $\mathrm{mg} / \mathrm{L}$, and was stable during the three years study [19].

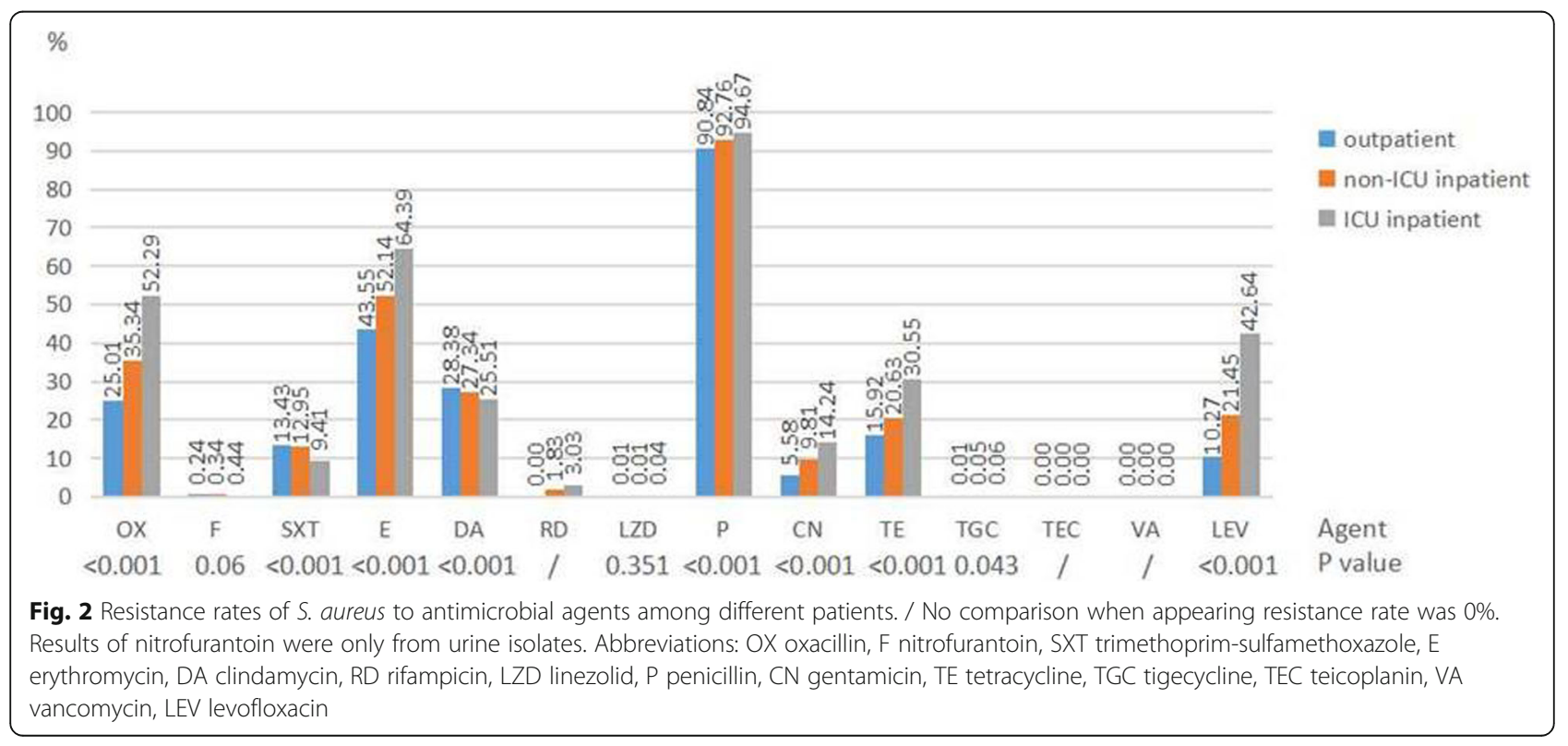




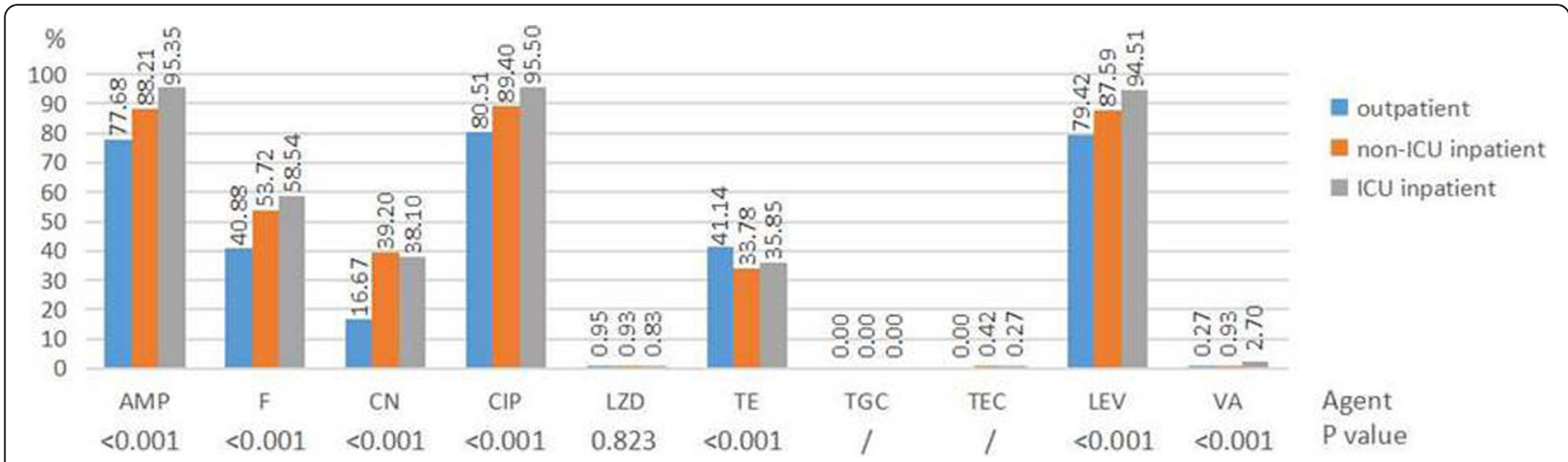

Fig. 3 Resistance rates of enterococci (E. faecalis and E. faecium) to antimicrobial agents among different patients. / No comparison when appearing resistance rate was $0 \%$. Results of nitrofurantoin, tetracycline, ciprofloxacin and levofloxacin were only from urine isolates. Abbreviations: AMP ampicillin, $\mathrm{F}$ nitrofurantoin, CN gentamicin-high level, CIP ciprofloxacin, LZD linezolid, TE tetracycline, TGC tigecycline, TEC teicoplanin, LEV levofloxacin, VA vancomycin

These results may be attributed to vancomycin being an unlikely empirical therapy due to its narrow-spectrum and its injection-only administration, thus resistances may not have had a chance to form. Meanwhile, vancomycin belongs to the highest limit level in the classification management system of antibiotics. Linezolid resistances in coagulase negative staphylococci are greater than resistance in S. aureus [20, 21]. Staphylococcus capitis isolates were the highest in Zhejiang province, and the resistance rates to linezolid increased from $1.8 \%$ in 2014 [20] to $3.5 \%$ in 2016 [21]. This is due to an outbreak of linezolid-resistant S. capitis infection in Zhejiang. Since the first report of linezolid resistance in methicillin-resistant coagulase negative staphylococci in the Second Affiliated Hospital of Zhejiang University School of Medicine in 2011, linezolid resistance in human clinical isolates has become an increasing problem in China [22, 23], and the cfr-carrying plasmid has appeared in S. aureus [24]. We isolated several linezolid-resistant $S$. aureus strains in 2016, but not in 2015 and 2017. No outbreaks of linezolid resistance were seen in S. aureus isolates.

The results of the present study show that the resistance rates of $E$. faecium are greater than the resistance rates of $E$. faecalis, and the resistance rates of E. faecium to ampicillin and quinolones are more than $80 \%$. Therefore infections caused by E. faecium present a serious clinical challenge for physicians [25], and treatment options for these infections are limited. Vancomycin is one treatment option that could be considered. In the last ten years, a weak downward trend for VRE cases was found worldwide and may be seen in the Zhejiang province. We also confirmed that the resistance rate of enterococcus to vancomycin remains at a low level, and showed downward trends, similar to other reports in China and lower than that in Ireland [26]. We found that the resistance rates of E. faecalis and E. faecium to vancomycin are higher than the resistance rates to teicoplanin. We speculate that strains with the dominant gene $v a n B$ may have the highest resistance to vancomycin [27]. These may have a different resistance than strains with the $v a n M$ gene, seen in China [28] or in strains with vanA dominance, seen in Poland [29]. In our study, resistance rate of E. faecalis to linezolid increased from $1.6 \%$ in 2008 to $2.97 \%$ in 2016 [21] and linezolid resistance was higher in E. faecalis than in $E$. faecium. In the past, mutations in the central loop of domain $\mathrm{V}$ of the $23 \mathrm{~S}$ rRNA represented the most common mechanism of oxazolidinone resistance in enterococci, with G2576 T (Escherichia coli numbering) as the predominant mutation [30]. Increasingly, transferable oxazolidinone resistance from the multi-resistance genes $c f r$ and optrA are being reported all over the world [31-33]. Furthermore, it was reported that the optrA gene was detected more frequently from food-producing animals than from humans [34].

In the multivariable analysis for risk factors associated with MRSA and VRE, we found that Hangzhou and Quzhou simultaneously had high MRSA and VRE detection rates. As the provincial capital of Zhejiang, Hangzhou has more tertiary hospitals and receives a greater number of critically ill patients from other cities. As a relatively under-developed city, Quzhou may have a poorer sanitary arrangement, and the compliance with antibiotics may be worse. Meanwhile, we found that the cities of relatively high MRSA and VRE detection rates tend to neighbour one another. This phenomenon may be caused by the increased likelihood of interaction between the populations and patients of these adjacent cities, allowing greater dissemination of MRSA and VRE isolates. To be a patient in a IIIA hospital (the highest classified and possibly largest hospital type in China) and a patient in the ICU ward are the greatest risk factors associated with MRSA and VRE infection. These findings are in accordance with the literature $[35,36]$ and can be attributed to patients with severe co-morbidities. Stratifying the data by patient age, it is observed that the proportion of MRSA and VRE was the highest in isolates 
Table 2 Analysis of risk factors associated with MRSA and VRE strains

\begin{tabular}{|c|c|c|c|c|}
\hline & \multicolumn{2}{|l|}{ MRSA } & \multicolumn{2}{|l|}{ VRE } \\
\hline & OR $(95 \% \mathrm{Cl})$ & $P$ value & OR (95\%Cl) & $P$ value \\
\hline \multicolumn{5}{|l|}{ Year } \\
\hline 2015 & $1.022(1.010-1.035)$ & $<0.001$ & $1.005(1.003-1.006)$ & $<0.001$ \\
\hline 2016 & $1.018(1.006-1.030)$ & 0.002 & 1.001 (0.999-1.003) & 0.214 \\
\hline 2017 & 1 & - & 1 & - \\
\hline \multicolumn{5}{|l|}{ Region } \\
\hline Hangzhou & $1.329(1.295-1.363)$ & $<0.001$ & $1.010(1.008-1.012)$ & $<0.001$ \\
\hline Huzhou & 1 & - & $1.025(1.017-1.033)$ & $<0.001$ \\
\hline Jiaxing & 1.109 (1.080-1.139) & $<0.001$ & $1.006(1.003-1.009)$ & $<0.001$ \\
\hline Jinhua & $1.229(1.195-1.265)$ & $<0.001$ & 1.005 (1.002-1.008) & 0.001 \\
\hline Lishui & $1.030(1.002-1.059)$ & 0.045 & $1(0.997-1.003)$ & 1 \\
\hline Ningbo & $1.406(1.365-1.447)$ & $<0.001$ & $1.002(1-1.004)$ & 0.054 \\
\hline Quzhou & $1.453(1.398-1.511)$ & $<0.001$ & $1.017(1.011-1.024)$ & $<0.001$ \\
\hline Shaoxing & 1.189 (1.156-1.224) & $<0.001$ & $1.003(1.001-1.006)$ & 0.015 \\
\hline Taizhou & $1.197(1.163-1.231)$ & $<0.001$ & 1.005 (1.002-1.009) & 0.001 \\
\hline Wenzhou & $1.282(1.245-1.319)$ & $<0.001$ & 1 & - \\
\hline Zhoushan & $1.775(1.676-1.880)$ & $<0.001$ & 1.004 (0.999-1.010) & 0.079 \\
\hline \multicolumn{5}{|l|}{ Hospital Level } \\
\hline IIIA $(n=46)$ & $1.267(1.213-1.322)$ & $<0.001$ & $1.016(1.009-1.024)$ & 0.031 \\
\hline$\| I I B(n=24)$ & $1.189(1.138-1.242)$ & $<0.001$ & 1.010 (1.002-1.019) & 0.143 \\
\hline$\| \mathrm{A}(n=15)$ & 1.139 (1.089-1.190) & $<0.001$ & $1.016(1.006-1.025)$ & 0.045 \\
\hline$\| \mathrm{B}(n=1)$ & 1 & - & 1 & - \\
\hline \multicolumn{5}{|l|}{ Age } \\
\hline$<=7 d$ & $1.130(1.063-1.201)$ & $<0.001$ & * & * \\
\hline $8 d-28 d$ & 1.085 (1.043-1.129) & $<0.001$ & * & * \\
\hline $1 \mathrm{~m}-1 \mathrm{y}$ & $1.054(1.030-1.079)$ & $<0.001$ & * & * \\
\hline $2 y-3 y$ & 1 & - & 1.008 (0.998-1.019) & 0.015 \\
\hline $4 y-19 y$ & $1.015(0.992-1.037)$ & 0.203 & 1 & - \\
\hline $20 y-43 y$ & $1.057(1.035-1.080)$ & $<0.001$ & $1.004(1.002-1.005)$ & 0.044 \\
\hline $44 y-59 y$ & $1.104(1.080-1.128)$ & $<0.001$ & 1.007 (1.005-1.009) & 0.002 \\
\hline $60 y-74 y$ & $1.190(1.164-1.217)$ & $<0.001$ & $1.006(1.004-1.008)$ & 0.004 \\
\hline $75+y$ & $1.443(1.409-1.478)$ & $<0.001$ & 1.011 (1.009-1.013) & $<0.001$ \\
\hline \multicolumn{5}{|l|}{ Specimen type } \\
\hline Blood & $1.142(1.101-1.185)$ & $<0.001$ & 1.007 (1.003-1.010) & $<0.001$ \\
\hline Bile & $1.385(1.151-1.665)$ & $<0.001$ & 1.001 (0.999-1.004) & 0.403 \\
\hline Respiratory & $1.318(1.278-1.360)$ & $<0.001$ & $1.003(0.995-1.011)$ & 0.515 \\
\hline Urine & $1.186(1.139-1.235)$ & $<0.001$ & $1.006(1.004-1.008)$ & $<0.001$ \\
\hline Secretion & $1.115(1.081-1.150)$ & $<0.001$ & 1 & - \\
\hline Puncture fluid & 1 & - & $1.004(1-1.009)$ & 0.012 \\
\hline stool & $1.342(1.262-1.426)$ & $<0.001$ & $1.001(0.997-1.005)$ & 0.839 \\
\hline Other & $1.137(1.102-1.173)$ & 0.002 & $1(0.998-1.002)$ & 1 \\
\hline \multicolumn{5}{|l|}{ Patient category } \\
\hline Outpatient & 1 & - & $1.004(0.995-1.014)$ & 0.4 \\
\hline Inpatient-non ICU & $1.132(1.118-1.147)$ & $<0.001$ & 1 & - \\
\hline
\end{tabular}


Table 2 Analysis of risk factors associated with MRSA and VRE strains (Continued)

\begin{tabular}{lllll}
\hline & MRSA & & VRE \\
\cline { 2 - 3 } & OR $(95 \% \mathrm{Cl})$ & $P$ value & OR (95\%Cl) & $P$ value \\
\hline Inpatient-ICU & $1.439(1.407-1.473)$ & $<0.001$ & $1.019(1.013-1.025)$ & $<0.001$ \\
\hline
\end{tabular}

OR odds ratio, $\mathrm{Cl}$ confidence interval

IIIA number of bed more than 500, comprehensive examination score more than 900 points

IIIB number of bed more than 500, comprehensive examination score between 750 and 899 points

IIA number of bed between 100 and 499, comprehensive examination score more than 900 points

IIB number of bed between 100 and 499, comprehensive examination score between 750 and 899 points

Comprehensive examination including departments, staffing, management level, technical level, work quality and technical facilities

$\mathrm{d}$ day old, $\mathrm{m}$ month old, $\mathrm{y}$ year old

Respiratory containing sputum and bronchoalveolar lavage fluid

Secretion containing pus and wound swab

Puncture fluid containing hydrothorax, ascites, articular cavity fluid, pericardial fluid and Cerebrospinal fluid

* No VRE isolate

from elderly patients older than 75 years, but was the lowest from children aged 2 years - 3 years and infants younger than 1 year. The high numbers of MRSA and VRE isolates from elderly patients may be due to these patients having more underlying diseases and a greater history of antibiotic use than the child and the infant group. As well, decreased nutrition and immune function, often seen in the elderly, may also be contributing elements. Other identified risk factors associated with MRSA and VRE include having a source of isolates. We found that puncture fluid (containing hydrothorax, ascites, articular cavity fluid, pericardial fluid and cerebrospinal fluid) has the lowest proportion $(21.32 \%)$ of MRSA, though the underlying reason for this phenomenon needs to be further studied. In terms of treatment, we should be wary of methicillin-sensitive $S$. aureus when $S$. aureus is isolated from puncture fluid in Zhejiang province. With the noted exception of blood and urine, there is no difference in the specimen types in proportions of VRE isolates.

These findings will provide valuable information for infection control practices. Although many surveillance projects of antibiotic resistance have been carried out in China, they always cover only the tertiary hospitals. In our study, a wider range of scenarios were seen where resistant strains could occur, indicating the importance of performing regional antibiotic resistance surveillance. The current study had some limitations: we had a relatively short span of time (3 years) for the data collection, we had a limited number of IIB hospitals to contribute data to the study, and because ours was a retrospective analysis, we had an inability to obtain the original strains. In the future, we would like to expand this surveillance to cover more IIB and rural area clinics and also collect the original strains for intensive study.

\section{Conclusion}

The detection rates of MRSA stayed at moderate levels, and VRE stayed at low levels during the last three years, and local dissemination was found in MRSA and VRE isolates. The highest risk factors for MRSA and VRE infection were patient status in a IIIA hospital, age older than 75 years and hospitalization in the ICU ward. As a result of our findings, we suggest that sustained surveillance is necessary to prevent the spread or clonal dissemination of drug-resistant strains in Zhejiang China.
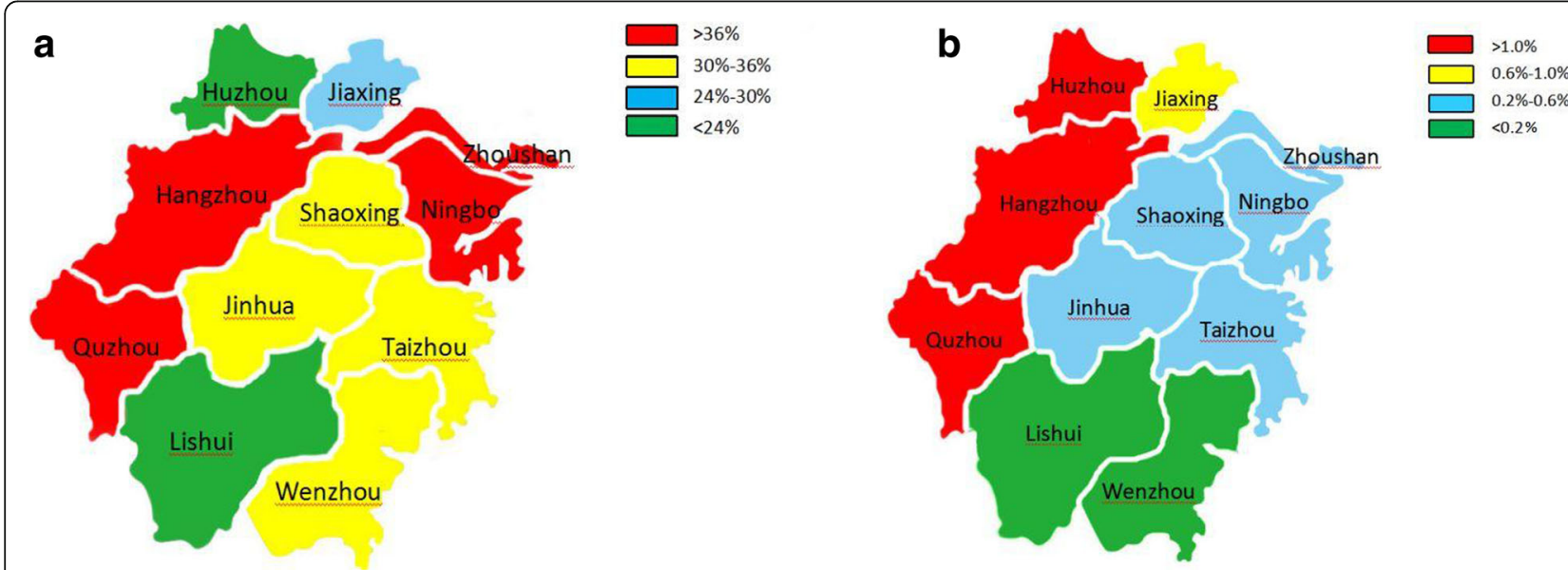

Fig. 4 Distribution of MRSA (a) and VRE (b) by geographic area 


\section{Abbreviations}

Cl: Confidence interval; ICU: Intensive care unit; MALDI-TOF-MS: MatrixAssisted Laser Desorption/ Ionization Time of Flight Mass Spectrometry; MIC: Minimum inhibitory concentration; MRSA: Methicillin-resistant Staphylococcus aureus; OR: Odds ratio; VRE: Vancomycin-resistant enterococci

\section{Acknowledgements}

We want to thank the participating hospitals for their work and cooperation. We also want to thank the drug resistance monitoring group of the Zhejiang Province.

\section{Funding}

This study was supported by grants from the National Natural Science Foundation of China (No. 81871705 from Gongxiang Chen).

\section{Availability of data and materials}

All data generated or analysed during this study are included in this manuscript.

\section{Authors' contributions}

HL, CJM, LHY, CS, DSB and ZR contributed to the acquisition and analysis of the data. HL wrote the initial draft of this paper. HYY and ZHW performed the statistical analyses. CGX contributed to the concept of the study, the revision of this paper, and the final approval of the version to be published. All authors have read and approved the final manuscript.

\section{Ethics approval and consent to participate}

Not applicable

\section{Consent for publication}

Not applicable

\section{Competing interests}

The authors declare that they have no competing interests.

\section{Publisher's Note}

Springer Nature remains neutral with regard to jurisdictional claims in published maps and institutional affiliations.

\section{Author details}

${ }^{1}$ Department of Clinical Microbiology, The Second Affiliated Hospital of Zhejiang University School of Medicine, 88 Jiefang Road, Hangzhou, Zhejiang 310009, People's Republic of China. 'Zhejiang Provincial Hospital of TCM, Hangzhou 310006, Zhejiang, China. ${ }^{3}$ Centre of Laboratory Medicine, Zhejiang Provincial People's Hospital, People's Hospital of Hangzhou Medical College, Hangzhou 310014, Zhejiang, China. ${ }^{4}$ Hangzhou Third Hospital, Hangzhou 310009, Zhejiang, China. ${ }^{5}$ Hangzhou Red Cross Hospital, Hangzhou 310003, Zhejiang, China.

\section{Received: 21 December 2018 Accepted: 13 May 2019} Published online: 30 May 2019

\section{References}

1. Bereket W, Hemalatha K, Getenet B, Wondwossen T, Solomon A, Zeynudin A, Kannan S. Update on bacterial nosocomial infections. Eur Rev Med Pharmacol Sci. 2012;16:1039-44.

2. Sacar S, Sayin KS, Turgut H, Cevahir N, Hircin Cenger D, Tekin K. Epidemiology and associated factors for nosocomial methicillin-resistant Staphylococcus aureus infection in a tertiary-care hospital. Epidemiol Infect. 2010;138(5):697-701.

3. DeLeo FR, Otto M, Kreiswirth BN, Chambers HF. Community-associated meticillin-resistant Staphylococcus aureus. Lancet. 2010;375:1557-68.

4. Uçkay I, Pires D, Agostinho A, Guanziroli N, Öztürk M, Bartolone P, Tscholl P, Betz M, Pittet D. Enterococci in orthopaedic infections: Who is at risk getting infected? Journal of Infection. 2017;75(4):309-14.

5. Peter $K$, Astrid M, Guido H, Michael B, Ludwig K, Stefan F, Wilfried P, Stephan E, Alois O, Lass-Flörl C, et al. Outbreak report: a nosocomial outbreak of vancomycin resistant enterococci in a solid organ transplant unit. Antimicrob Resist Infect Control. 2018;7(1):86.

6. Rupp ME, Fitzgerald T, Hayes K, Van ST, Hewlett A, Clevenger R, Lyden E. Effect of cessation of contact isolation for endemic methicillin-resistant
Staphylococcus aureus and Vancomycin-resistant Enterococci. Infect Control Hosp Epidemiol. 2017;38(8):1-3.

7. Ramirez MJY, Nick D, Elias MN, Amuah JE, Kathryn B, Couris CM, Kira L. A comparison of administrative data versus surveillance data for hospitalassociated methicillin-resistant Staphylococcus aureus infections in Canadian hospitals. Infect Control Hosp Epidemiol. 2017;38(4):436-43.

8. Watson S, Cabrera-Aguas M, Khoo P, Pratama R, Gatus BJ, Gulholm T, El-Nasser J, Lahra MM. Keratitis antimicrobial resistance surveillance program, Sydney, Australia: 2016 Annual Report. Clin Exp Ophthalmol. 2019:47(1):20-5.

9. Seas C, Garcia C, Salles MJ, Labarca J, Luna C, Alvarez-Moreno C, MejíaVillatoro C, Zurita J, Guzmán-Blanco M, Rodríguez-Noriega E, et al. Staphylococcus aureus bloodstream infections in Latin America: results of a multinational prospective cohort study. J Antimicrob Chemother. 2018;73(1).

10. Zhang GS, Lin XH, Cai JC, Zhang R, Xiu HQ, Tian BP, Cui W. Characteristics of clinical and environmental, vanM-carrying vancomycin-resistant enterococci isolates from an infected patient. Int J Antimicrob Agents. 2018: S0924857918300062

11. Zhao C, Sun H, Wang H, Liu Y, Hu B, Yu Y, Sun ZY, Chu YZ, Cao B, Liao K, et al. Antimicrobial resistance trends among 5608 clinical gram-positive isolates in China: results from the gram-positive Cocci resistance surveillance program (2005-2010). Diagn Microbiol Infect Dis. 2012;73(2):174-81.

12. Sun HL, Wang H, Chen MJ, Liu YM, Hu ZD, Liao K, Chu YZ, Lei JE, Zhang B, Yu YS, et al. An antimicrobial resistance surveillance of gram-positive cocci isolated from 12 teaching hospitals in China in 2009. Chin J Lab Med. 2006; 49(9):735.

13. Jorgensen $\mathrm{JH}$. Manual of clinical microbiology, 11th edition [J]; 2015.

14. Patel JB, Cockerill lii FR, Alder J. Performance standards for antimicrobial susceptibility testing; twenty-fourth InformationalSupplement. Clinical and laboratory standards institute antimicrobial susceptibility testing M100-S24, vol. 34; 2014. p. 1-230.

15. Decousser JW, Woerther PL, Soussy CJ, Marguerite FG, Dowzicky MJ. The tigecycline evaluation and surveillance trial; assessment of the activity of tigecycline and other selected antibiotics against gram-positive and gramnegative pathogens from France collected between 2004 and 2016. Antimicrob Resist Infect Control. 2018:7(1):68.

16. European Centre for Disease Prevention and Control. Surveillance of antimicrobial resistance in Europe 2016. Annual report of the European antimicrobial resistance network (EARS-net). Stockholm: ECDC; 2017.

17. Shibl AM, Memish ZA, Kambal AM, Ohaly YA, Ishaq A, Senok AC, Livermore DM. National surveillance of antimicrobial resistance among gram-positive bacteria in Saudi Arabia. J Chemother. 2014;26(1):13-8.

18. Marco F, Dowzicky MJ. Antimicrobial susceptibility among important pathogens collected as part of the Tigecycline evaluation and surveillance trial (T. E.S.T.) in Spain, 2004-2014. J Glob Antimicrob Resist. 2016;6:50-6.

19. Kehrmann J, Kaase M, Szabados F, Gatermann SG, Buer J, Rath PM, Steinmann J. Vancomycin MIC creep in MRSA blood culture isolates from Germany: a regional problem? Eur J Clin Microbiol Infect Dis. 2011;30(5):677-83.

20. Xie XY, Yu YS. Zhejiang provincial hospital bacterial resistance testing yearbook. 2015. Zhejiang University Press; 2015.

21. Xie XY, Yu YS. Zhejiang provincial hospital bacterial resistance testing yearbook. 2017. Zhejiang University Press; 2017.

22. Yang XJ, Chen Y, Yang Q, Qu TT, Liu LL, Wang HP, Yu YS. Emergence of cfrharbouring coagulase-negative staphylococci among patients receiving linezolid therapy in two hospitals in China. J Med Microbiol. 2013;62:845-50.

23. Cui L, Wang Y, Li Y, He T, Schwarz S, Ding Y, Shen J, LV Y. Cfr-mediated linezolid-resistance among methicillin-resistant coagulase negative staphylococci from infections of humans. PLoS One. 2013;8(2):e57096.

24. Cai JC, Hu YY, Zhou HW, Chen GX, Zhang R. Dissemination of the same cfrcarrying plasmid among methicillin-resistant staphylococcus aureus and coagulase-negative staphylococcal isolates in China. Antimicrob Agents Chemother. 2015:59(6):3669-71.

25. Gudiol C, Ayats J, Camoez M, Domínguez MA, García-Vidal C, Bodro M, Ardanuy C, Obed M, Arnan M, Antonio M, et al. Increase in bloodstream infection due to Vancomycin-susceptible Enterococcus faecium in Cancer patients: risk factors, molecular epidemiology and outcomes. PLoS One. 2013;8(9):e74734.

26. Morris-Downes M, Smyth EG, Moore J, Thomas T, Fitzpatrick F, Walsh J, Caffreyc V, Morrisc A, Foleye S, Humphreys $H$. Surveillance and endemic vancomycin-resistant enterococci: some success in control is possible. J Hosp Infect. 2010;75(3):228-33. 
27. Zhou X, Arends JP, Kampinga GA, et al. Evaluation of the Xpert vanA/vanB assay using enriched inoculated broths for direct detection of vanB vancomycin-resistant Enterococci.[J]. J Clin Microbiol. 2014;52(12):4293.

28. Chen CH, Sun JY, Guo Y, Lin DF, Guo QL, Hu FP, Zhu DM, Xu XG, Wang MG. High prevalence of vanM in vancomycin-resistant Enterococcus faecium isolates from Shanghai, China. Antimicrob Agents Chemother. 2015;59(12):7795-8.

29. Wardal E, Kuch A, Gawryszewska I, Żabicka D, Hyniewicz W, Sadowy E. Diversity of plasmids and Tn 1546 -type transposons among VanA Enterococcus faecium, in Poland. Eur J Clin Microbiol Infect Dis. 2017;36(2):313-28.

30. Arias CA, Murray BE. Emergence and management of drug-resistant enterococcal infections. Expert Rev Anti-Infect Ther. 2008;6:637-55.

31. Diaz L, Kiratisin P, Mendes RE, Panesso D, Singh KV, Arias CA. Transferable plasmid-mediated resistance to linezolid due to $\mathrm{cfr}$ in a human clinical isolate of Enterococcus faecalis. Antimicrob Agents Chemother. 2012;56(7):3917-22.

32. Zhang YZ, Dong GF, Li YH, Chen LJ, Liu HY, Bi WZ, Lu H, Zhou TL. A high incidence and coexistence of multiresistance genes cfr and optrA among linezolid-resistant enterococci isolated from a teaching hospital in Wenzhou, China. Eur J Clin Microbiol Infect Dis. 2018;37(8):1441-8.

33. Lazaris A, Coleman DC, Kearns AM, Pichon B, Kinnevey PM, Earls MR, Boyle B, O'Connell B, Brennan Gl, Shore AC. Novel multiresistance cfr plasmids in linezolid-resistant methicillin-resistant Staphylococcus epidermidis and vancomycin-resistant Enterococcus faecium (VRE) from a hospital outbreak: COlocation of cfr and optrA in VRE. J Antimicrob Chemother. 2017;72(12):3252-7.

34. Wang Y, Lv Y, Cai JC, Schwarz S, Cui L, Hu Z, Zhang R, Li J, Zhao Q, He T, et al. A novel gene, optrA, that confers transferable resistance to oxazolidinones and phenicols and its presence in Enterococcus faecalis and Enterococcus faecium of human and animal origin. J Antimicrob Chemother. 2015;70(8):2182-90.

35. Upreti N, Rayamajhee B, Sherchan SP, Choudhari MK, Banjara MR. Prevalence of methicillin resistant Staphylococcus aureus, multidrug resistant and extended spectrum $\beta$-lactamase producing gram negative bacilli causing wound infections at a tertiary care hospital of Nepal. Antimicrob Resist Infect Control. 2018;7(1).

36. Zhou XW, Garcia-Cobos S, Ruijs GJHM, Greetje A, Kampinga AJP, Bors DM, Moller LV. Epidemiology of extended-Spectrum beta-lactamase-producing E-coli and Vancomycin-resistant Enterococci in the northern Dutch-German cross-border region. Front Microbiol. 2017;8.

Ready to submit your research? Choose BMC and benefit from:

- fast, convenient online submission

- thorough peer review by experienced researchers in your field

- rapid publication on acceptance

- support for research data, including large and complex data types

- gold Open Access which fosters wider collaboration and increased citations

- maximum visibility for your research: over $100 \mathrm{M}$ website views per year

At $\mathrm{BMC}$, research is always in progress.

Learn more biomedcentral.com/submissions 